\title{
Performance of an Endian Operating System for Step Topology
}

\author{
Taskeen Zaidi \\ Research Scholar, Deptt. of Computer Science \\ Babasaheb Bhimrao Ambedkar University \\ Vidya Vihar, Rae Bareli, Lucknow
}

\author{
Vipin Saxena \\ Professor, Deptt. of Computer Science \\ Babasaheb Bhimrao Ambedkar University \\ Vidya Vihar, Rae Bareli Road, Lucknow
}

\begin{abstract}
In the current scenario, distributed computing approach is most popular approach for computing the processes known as subtasks, subroutines, macro's etc due to low cost involvement and minimum execution time. In the present work, the computer systems are attached with the help of static interconnection through step topology in which one machine is working as server and Endian operating system is loaded on the server. A Unified Modeling Language (UML) is used to demonstrate the model of working of Endian operating system. The performance of Endian operating system in terms of firewall execution is monitored for the various user identification controlled connected by Internet Protocol (IP Version 4) where the users are connected through static topology. The loading and balancing are also performed by Endian operating system for transferring of data from one machine to another machine and results are depicted in terms of tables and graphs.
\end{abstract}

\section{Keywords}

Distributed approach, Endian operating system, step topolgy, IP address.

\section{INTRODUCTION}

In the past few years, distributed computing approach is the most popular approach due to several advantages over the centralized approach. In this approach different kinds of the devices like laptop's, desktop's, mobile devices, etc. can be connected and enjoy the benefits of sharing of the resources although they do not share the global clock. All kinds of the computer networks are well described in Tanenbaum[1] which contains the whole generation of computer network, network topologies, adhoc networks, communication styles and secure transmission of data with special emphasis on wireless networking. In this regard, another important reference is Forouzan[2] which has described the security features when user is attached to the internet i.e. IP security, SSL/TLS, VPN, PGP, firewalls and network security. In the year 2009, India has launched the National Knowledge Network (NKN) under the Next Generation Network (NGN) services to connect the various higher educational Institutions, Universities, research labs, libraries and cloud computing centres. In this regard, Gloria et al.[3] has explained the establishment and significance of NKN which connects all kinds of research labs, medical labs and enhanced e-learning. The concept of establishment of NKN is well explained in [4] which explained the services, applications, list of connected Institutions, design and architecture of NKN and current status of NKN project. All the services provided by NKN are also well explained in[5,6] including voice services which is provided by Railtel India Corporation, New Delhi, India. The link has the support upto 20Gbbps data transfer and currently various Institutions connected under NKN are enjoying $1 \mathrm{Gbps}$ data transferring speed over the internet. Recently Saxena et al.[7] have described the role of NKN for accessing the information using Information Library Network (INFLIBNET) which will enhance the quality of teaching and research in improving the quality of higher education through E-learning. Cisco is the brand of all devices connected under NKN[8] and all these devices are installed by Hindustan Corporation Limited (HCL) which provides secure and reliable connectivity of the National Knowledge etwork in India. The significance of NKN is to enhance the quality of teaching and research in higher education and to promote Elearning rather than using the traditional approach and these are well explained in [9-12]. When the users are working in the heterogeneous system, the security of information is essential over the network in which all kinds of the users are attached through the network. The security threats caused by the users and providers are well explained by Milenkovic[13]. From time to time, different types of the topologies have appeared in the literature for the static and dynamic interconnections of computer systems. These are namely star, tree bus, ring, mesh, hyper cube, etc. and these are well summarized by Hwang[14].

In the past year, it is observed from the literature that the modeling aspects are also applied in the field of distributed computing. A well known Unified Modeling Language (UML) aspect is also used by the various researchers for modeling of the research problems related to the software and hardware as the modeling supports the object-orientation. Let us describe some of the important references related to the modeling aspects. The XML metadata specification is presented using UML diagrams by Object Management Group (OMG) which are well explained in [15- 16]. The UML modeling design issues related to distributed and parallel applications has explained in [17-18]. Recently, Saxena and Zaidi [19] has also proposed a step topology for static interconnections of computer systems under distributed environment in which $\mathrm{N}$ number of computer systems are connected by varying cable segments through a new kind of topology called as step topology. The significance of NKN project, advantages and difficulties faced by this project and a case study of percentage utilization of NKN project has been studied by Saxena and Zaidi [20]. UML modeling for finding the performance of different topologies have been described by Saxena et al.[21]. A new protocol to establish mutual exclusion in distributed system using bidirectional ring is designed by Saxena and Arora [22].

In the present work, a performance of static interconnection has been observed over the National Knowledge Network in which the computer systems are attached with the help of a new designed topology called as step topology. The performance for executing the processes over the step topology has been observed through the working of the well known Endian operating system which has been loaded on the server. The different users are attached through the computer 
systems connected by step topology. Authors have evaluated the percentage utilization, elapsed time and bytes transferred from one computer system to another computer system and computed results are depicted in the form of tables and graphs.

\section{BACKGROUND}

\subsection{A Distributed System}

In the recent years, distributed computing system is very popular for computing the processes, subtasks, subroutines, macros etc., due to its capability to deliver high end performance computing at a very low cost. The distributed system consists of multiple computers or devices called as nodes which are interconnected by message passing technique of network. The devices are interacted with the help of message passing techniques in distributed system. A network of interconnection of these systems is called as National Knowledge Network (NKN) which is implemented under Next Generation Network (NGN) services in India. A representation of distributed system is shown in fig. 1 .

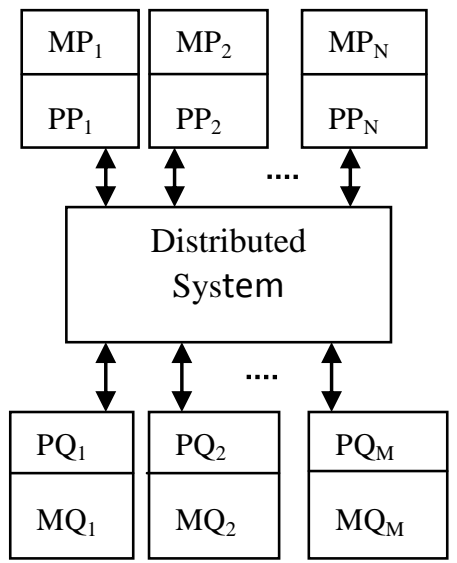

Fig 1: A distributed system

In the above diagram $\mathrm{PP}_{1}, \mathrm{PP}_{2}, \ldots, \mathrm{PP}_{\mathrm{N}}$ and $\mathrm{PQ}_{1}, \mathrm{PQ}_{2}, \ldots, \mathrm{PQ}_{\mathrm{M}}$ are the different processors having different processing speed while $\mathrm{MP}_{1}, \mathrm{MP}_{2}, \ldots, \mathrm{MP}_{\mathrm{N}}$ and $\mathrm{MQ}_{1}, \mathrm{MQ}_{2}, \ldots, \mathrm{MQ}_{\mathrm{M}}$ are the different memories attached to the corresponding processors as shown in the above figure.

\subsection{Endian Operating System}

This kind of operating system is used to handle the amount of large data stored in the external memory under distributed computing environment. Large database are stored in the memory and fragmented into the smaller pieces for execution purpose and later on concatenated the pieces of the data. The system installed on the server has the following configuration.

\begin{tabular}{|l|}
\hline Configuration of Endian Operating System \\
Free BSD on MIPS, ARM, PowerPC and SPARC \\
HP-UX on Itanium and PA-RISC \\
Linux on MIPS, SPARC,PS-RISC, PowerPC, \\
680x0,ESA/390,z/Architecture, H8, FR-V,AVR32, \\
ARMEB,M32R,SHEB, Ub icom32
\end{tabular}

\author{
Mac Operating System \\ NetBSD on Power PC, SPARC,SGI,Motorola 68k and \\ $88 \mathrm{k}$ \\ MVS and DOS/VSE on ESA/390 and z/VSE and z/OS \\ and $\mathrm{z} /$ Architecture
}

Solaris on SPARC

The above operating system contains the z/Architecture which is most powerful in respect of other architectures in terms of security of large amount of database routed from one computer system to other computer system. In the proposed system, Endian operating system is loaded on the server and all the database is routed through this server and the machines are arranged with the help of the step topology and performance of this operating system is observed and it is found that it is most powerful security system for handling the large amount of database.

\subsection{Topologies}

From time to time various researchers have proposed different types of the static interconnection of computer systems. The most popular static interconnections are the linear array, ring, tree, star which are widely used for setting up the computer labs. Recently and due to evolution of multithreaded system number of researchers are using extension of mesh topology which supports $\mathrm{N} x \mathrm{~N}$ number of computer systems. The other categories of the topologies are fat tree, barrel shifter, systolic array, hypercube etc. These are well defined in the literature with space complexity and currently used for connection of number of devices connected under distributed computing systems. Recently authors have developed a new kind of static interconnection called as a step topology by considering the concept of staircases. By using this topology, $\mathrm{N}$ number of computer system can be connected under distributed computing system. In the present work, performance of the well know Endian operating system is observed for the $\mathrm{N}$ computer systems which are attached through step topology.

\subsection{Critical section}

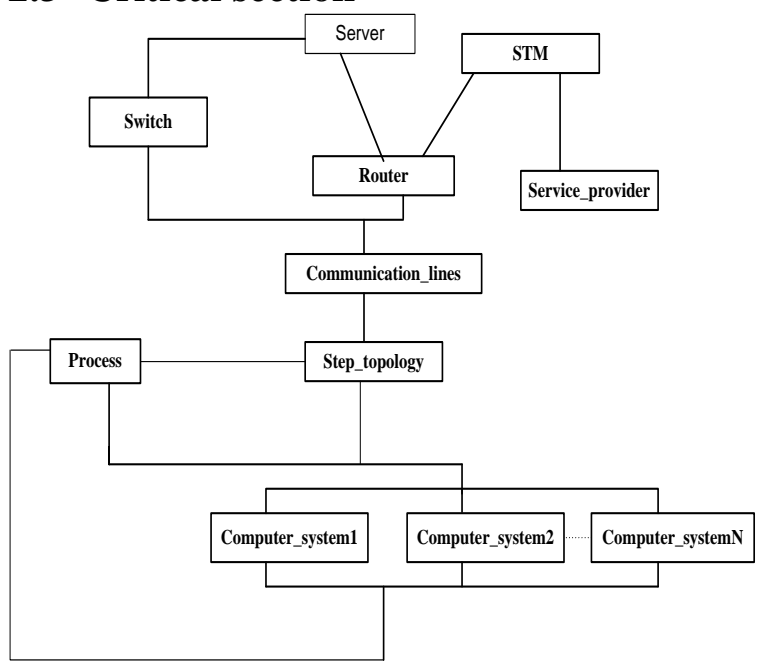

Fig 2: Execution of process under step topology 
In the Endian operating system, the number of computer systems are attached through step topology and under NGN services and a nationwide network has been established by connecting the classes namely Service_provider, STM, Router, Server and layer 3 category of Switch. Finally these classes are attached with the help of another class called as Communication_lines through which step topology has been implemented. The segment of step topology is shown below:

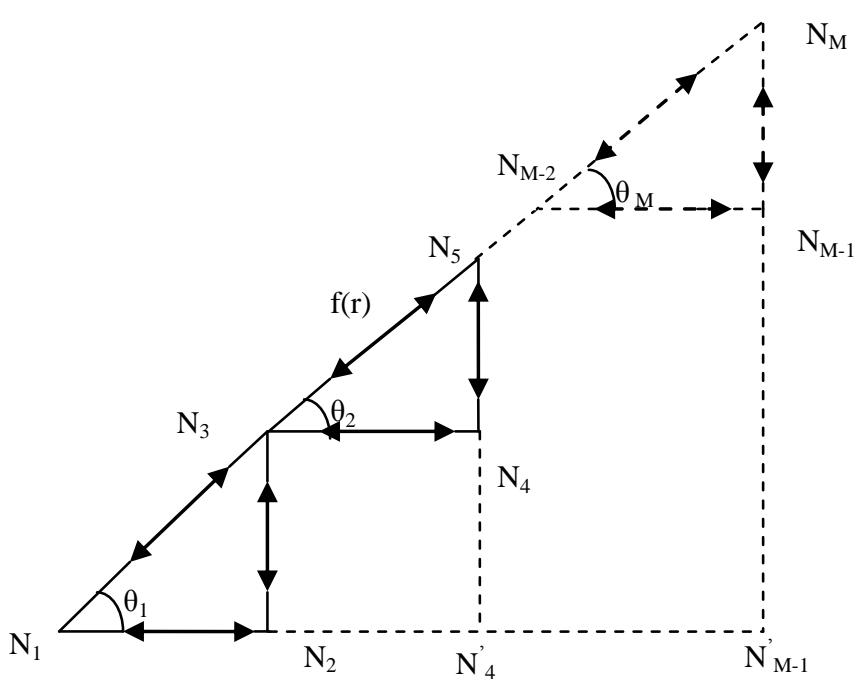

Fig 3: Representation of step topology

The step topology is defined as:

For the $\mathrm{N}_{\mathrm{M}}$ computer systems, consider the length of segments as described below:

$$
\begin{aligned}
& \mathrm{N}_{1} \mathrm{~N}_{2}=\alpha_{1}, \mathrm{~N}_{1} \mathrm{~N}_{4}^{\prime}=\alpha_{2}, \ldots \ldots \ldots \ldots \mathrm{N}_{1} \mathrm{~N}_{\mathrm{M}-2}^{\prime}=\alpha_{\mathrm{M}-1}, \mathrm{~N}_{1} \mathrm{~N}_{\mathrm{M}-1}^{\prime}=\mathrm{r} \\
& \beta_{1}=\tan \theta_{1}, \beta_{2}=\tan \theta_{2}, \ldots \ldots \ldots \ldots \ldots \ldots \ldots \beta_{\mathrm{M}}=\tan \theta_{\mathrm{M}}
\end{aligned}
$$

$\mathrm{f}(\mathrm{r})$ is the radial distance measuring the diameter of the step topology as described below:

$$
f(r)=1+\beta_{1} r+\beta_{2}\left(r-\alpha_{1}\right)+\beta_{3}\left(r-\alpha_{2}\right)+\ldots \ldots \ldots+\beta_{M}\left(r-\alpha_{M-1}\right) .
$$

where, $r$ is the horizontal distance of the cable controlling the computer systems and measured in meters, while $\alpha_{1}, \alpha_{2}$, .......... $=\alpha_{\mathrm{M}-1}$ are the cable segments as shown in the above figure. In the above diagram, under distributed computing system, process can be executed on either node and the resultant can be displayed either of computer system or can be stored inside the file called as process. The systems are controlled through IP address by using the message passing technique.

\section{EXPERIMENTAL STUDY}

As per the figure 3, an experimental study has been performed and observed the study of pattern of the users by storing the report of the users. The report is generated for a period of twenty four hours. In this study, a real case study of Higher learning Institution namely Babasaheb Bhimrao Ambedkar University, Lucknow, India is considered and observed performance for sample size of four hundred computer systems but due limitation of space it is summarized below for ten computer systems. The data for ten computer systems is extracted from the four hundred computer systems.

\subsection{Bytes transferred}

The following table shows Endian firewall HTTP Proxy access report for the total bytes transferred by the users. The users are controlled by the Internet Protocol version 4 as shown in the table.

Table 1. IP vs Bytes Transferred

\begin{tabular}{|c|c|c|}
\hline Sl. No. & Userid & Bytes \\
\hline 1 & 192.168 .70 .44 & $10.83 \mathrm{G}$ \\
\hline 2 & 192.168 .70 .131 & $3.30 \mathrm{G}$ \\
\hline 3 & 192.168 .70 .67 & $3.07 \mathrm{G}$ \\
\hline 4 & 192.168 .70 .125 & $3.04 \mathrm{G}$ \\
\hline 5 & 192.168 .40 .30 & $2.97 \mathrm{G}$ \\
\hline 6 & 192.168 .70 .98 & $2.88 \mathrm{G}$ \\
\hline 7 & 192.168 .40 .26 & $2.88 \mathrm{G}$ \\
\hline 8 & 192.168 .70 .4 & $2.59 \mathrm{G}$ \\
\hline 9 & 192.168 .70 .6 & $2.40 \mathrm{G}$ \\
\hline 10 & 192.168 .70 .136 & $2.19 \mathrm{G}$ \\
\hline
\end{tabular}

From the above table it is concluded that the top user having IP address 192.168.70.44 transferred maximum bytes of 10.83GB and user having IP 192.168.70.136 transferred minimum bytes $2.19 \mathrm{~GB}$. It is observed that the data is related to the multimedia images transferred from one computer system to another computer system through internet. The graphical view of the above table is also shown below in fig. 4.

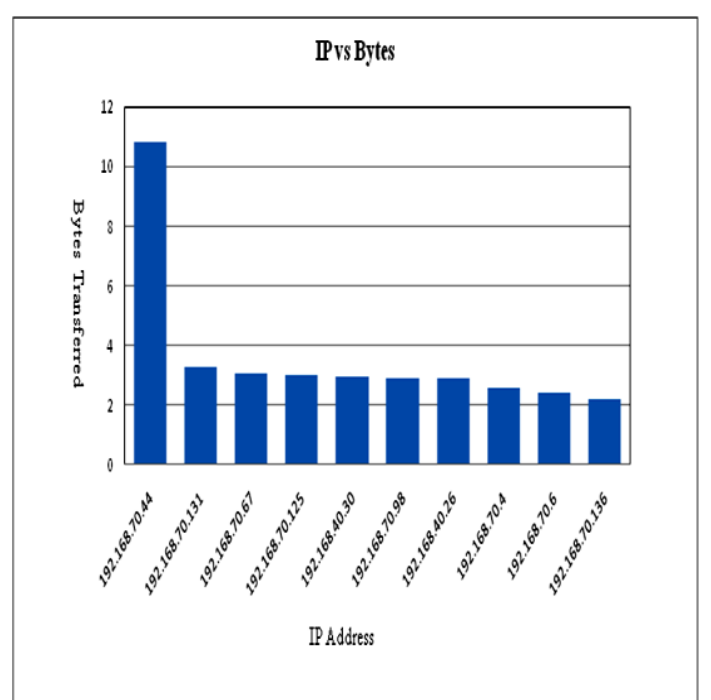

Fig 3: Representation of Bytes Transferred 


\subsection{Computed elapsed time}

Elapsed time is the time required to execute the process. From the generated report, the total elapsed time required by each user is recorded in millisecond which is given below in following table.

Table 2. IP vs Elapsed Time

\begin{tabular}{|c|c|c|}
\hline Sl. No. & User_id & Millisecond \\
\hline 1 & 192.168 .70 .44 & $51.35 \mathrm{~m}$ \\
\hline 2 & 192.168 .70 .131 & $42.02 \mathrm{~m}$ \\
\hline 3 & 192.168 .70 .67 & $52.50 \mathrm{~m}$ \\
\hline 4 & 192.168 .70 .125 & $96.00 \mathrm{~m}$ \\
\hline 5 & 192.168 .40 .30 & $29.12 \mathrm{~m}$ \\
\hline 6 & 192.168 .70 .98 & $55.45 \mathrm{~m}$ \\
\hline 7 & 192.168 .40 .26 & $82.40 \mathrm{~m}$ \\
\hline 8 & 192.168 .70 .4 & $29.52 \mathrm{~m}$ \\
\hline 9 & 192.168 .70 .6 & $36.90 \mathrm{~m}$ \\
\hline 10 & 192.168 .70 .136 & $66.95 \mathrm{~m}$ \\
\hline
\end{tabular}

From the above table it is concluded user having IP address 192.168.70.125 takes maximum elapsed time 96.00 milliseconds and user having IPaddress192.168.40.30 takes minimum elapsed time 29.12 milliseconds. It is also observed that these users were involved for downloading the video's from the internet. Since the bandwidth connectivity is of 1 Gbps therefore it is found that a large file containing the large database can be downloaded within fraction of milliseconds. The graphical representation is also shown below:

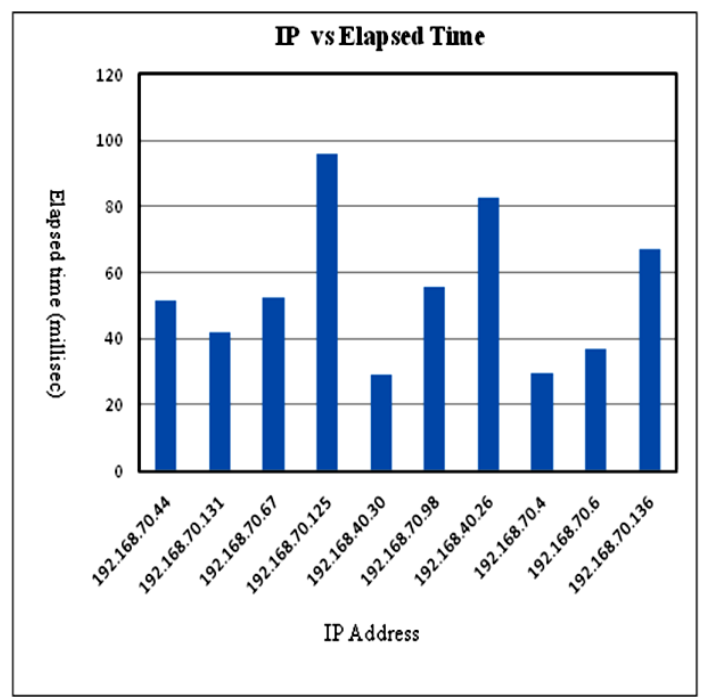

Fig 4: Representation of Elapsed Time

\subsection{Processor utilization}

The processor utilization is an important activity which shows the performance of the machine. It shows the number of processes out after the completion of execution in a particular time period. The Endian operating system has observed the processor utilization for the top users as recorded below in the following table.

Table 3. IP vs \% Utilization

\begin{tabular}{|c|c|c|}
\hline Sl. No. & User_id & \%Utilization \\
\hline 1 & 192.168 .70 .44 & $19.82 \%$ \\
\hline 2 & 192.168 .70 .131 & $64.89 \%$ \\
\hline 3 & 192.168 .70 .67 & $69.82 \%$ \\
\hline 4 & 192.168 .70 .125 & $70.52 \%$ \\
\hline 5 & 192.168 .40 .30 & $72.09 \%$ \\
\hline 6 & 192.168 .70 .98 & $74.38 \%$ \\
\hline 7 & 192.168 .40 .26 & $74.43 \%$ \\
\hline 8 & 192.168 .70 .4 & $82.82 \%$ \\
\hline 9 & 192.168 .70 .6 & $89.38 \%$ \\
\hline 10 & 192.168 .70 .136 & $93.74 \%$ \\
\hline
\end{tabular}

From the above table it is concluded that the user having IP address 192.168.70.136 has maximum utilization and user having IP address 192.168.70.44 has minimum utilization. The above data is also shown below in the form of the graph represented in the fig. 4 .

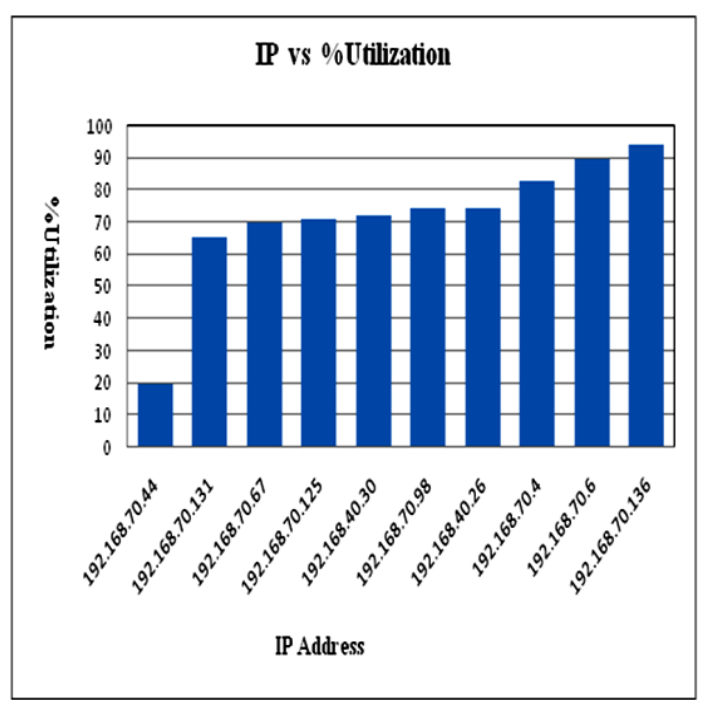

Fig 4: Representation of \% Utilization

\section{CONCLUSIONS}

From the above work, it is concluded that Endian operating system is a powerful operating system for keeping the records of the users attached on the computer networks. It creates systematic statistics for all the users in terms of the bytes transferred, usage of space, turnaround time taken by the users and overall utilization of the machines attached on the network. In the above work, a powerful modeling language is used to model the role of critical section used for the execution of the process. The computed results are efficient and real data is observed for the higher learning educational University attached through the National Knowledge Network under Next Generation Services. Since the Endian operating 
system generates the data in bulk, therefore for tracking purpose or to find the unauthorized access of the network, it can be represented in future in the form of the data cubes where mining techniques can be implemented to extract the desired information in a fraction of milliseconds. The comparison among the IP's address in terms of processor utilization, elapsed time and bytes transferred are shown in the above tables and graphs which shows the minimum and maximum boundaries of data.

\section{ACKNOWLEDGMENTS}

The authors are very thankful to University Grants Commission for providing financial assistance to the Department of Computer Science, Babasaheb Bhimrao Ambedkar University, Lucknow to carry out the above research work.

\section{REFERENCES}

[1] Andrew S.Tanenbaum., "Computer Networks", Prentice Hall, 2010.

[2] Frouzen, B. A., "Data Communications and Networks", Tata McGraw Hill, 2006.

[3] Gloria, C. Alaneme, Peter O. Olayinola, Comfort O.Raju, "Countering Traditional Learning and Elearning Methods in Higher Distance Education:Assessing Learness Preference", $4^{\text {th }}$ International Conference on Distance Education (ICDLE), 187-190,2010

[4] www.nkn.in"Fundamentals of National Knowledge Network" (Accessed on 12 ${ }^{\text {th }}$ Aug.2012).

[5] www.wikipedia.org "Introduction to National Knowledge network”(Accessed on 24 Aug.2012).

[6] www.railtelIndia.com/Infrastructure.aspx "Kinds of services for National Knowledge Network"(Accessed on $26^{\text {th }}$ Aug.2012).

[7] Saxena,V., Chaurasia, P.K. and Mishra, N., "E-Learning through National Knowledge Network", International Journal of Computer \& Electrical Engineering", Vol3(4), 554-556, 2011.

[8] www. cisco.com "Information about devices for National Knowledge Network" (Accessed on $5^{\text {th }}$ Sep.2012).

[9] Zhang, N. and Bao, H., "Research on the Computer Technology for E-Learning in Higher Education", International Conference on E-Education, E-Business, EManagement and E-Learning 295-298, 2010.

[10] Zhang, N. and Bao, H., "Research on E-Learning with Technology in Distance Education", International
Conference on e-Education, e-Business, e-Management and e-Learning 299-302, 2010.

[11] Jiong, Y. and Ting, L., "E-Learning in University:A Social Psychology Perspective", International Conference on E- Business and E-Government, 51445147,2010

[12] Nyarko, M., and Venture, N., "E-Learning:Virtual Cleanrooms as an Added learning Platform", IEEE region 8 SIBIRGN, July11-15, 426-431, 2010.

[13] Milenkovic, M.,Operating Systems:Concepts and Design, Tata McGraw Hill, 1987.

[14] Hwang, K. Advanced Computer Architecture: Parallelism, Scalability, Programmability, Fourteen reprint, Tata MCGraw Hill Edition, 2007, ISBN-0-07, 053070 .

[15] OMG,Unified Modeling Language Specification. Available online via http://www.omg.org,2001

[16] OMG, "Unified Modeling Language (UML)-Version 1.5", OMG document formal/2003-3-01, (2003), Needham MA.

[17] Pllana, S. and Fahringer, T. On Customizing the UML for Modeling Performance Oriented Applications. In $<<$ UML $>>$, Model Engineering Concepts and Tools, Springer-Verlag., Dresden, Germany,2002.

[18] Pllana, S.and Fahringer, T. UML Based Modeling of Performance Oriented Parallel and Distributed Applications, Winter Simulation Conference,2002.

[19] Saxena, V. and Zaidi, T., Step Topology for Static Interconnection of Computer Systems under Distributed Environment,WCSIT conference 2012.

[20] Saxena, V. and Zaidi , T.,National knowledge Network vs Information Communication Technology, University Department of Mathematics ,B.R.A. Bihar University,Muzzafarfur,India,11-12 feb,2012.

[21] Saxena, V., Arora, D. and Ahmad, S.,"ObjectOriented Distributed Architecture System through UML", Conference IEEE, International Conference on Advances in Computer Vision and Information Technology, ACVIT-07, ISBN 978-81-89866-74-7, pp.305-310,2007.

[22] Saxena, V. and Arora, D., 2008 "UML Modeling of a Protocol for Establishing Mutual Exclusion in Distributed Computer System", International Journal of Computer Science and Network Security,Vol.8, No.6, pp.227-235. 